\title{
Investigation of Genetic Variants and Causal Biomarkers Associated with Brain Aging
}

Jangho Kim, Junhyeong Lee, and Seunggeun Lee

Graduate School of Data Science, Seoul National University, Republic of Korea

Corresponding Author:

Seunggeun Lee

Graduate School of Data Science

Seoul National University, Seoul, South Korea

lee7801@snu.ac.kr

\begin{abstract}
Delta age is a biomarker of brain aging that captures differences between the actual age and the predicted age from brain MRI. This study investigates an explainable and causal basis of high delta age by analyzing UK Biobank data in three steps. A visual saliency map of brain regions showed that lower volumes in the fornix and the lower part of the thalamus are key predictors of high delta age. Genetic association analysis on both the single variants and the gene regions identified that genes related to carcinogenesis, immune response, and neuron survival are associated with high delta age. Mendelian randomization (MR) for all metabolomic biomarkers and blood-related phenotypes showed that immunerelated phenotypes have a causal impact on increasing delta age. Taken together, these observations revealed regions in the brain that are susceptible to the aging process and provided evidence of the causal and genetic connection between immune responses and brain aging.
\end{abstract}

Keyword : Delta age, Brain aging, Causality, CNN, Explainable AI, GWAS, Mendelian randomization, Immunity 


\section{Introduction}

Aging is a primary risk factor for a myriad of health problems. Since aging proceeds at different rates for each individual, various methods to measure the biological age have been developed to make a more accurate diagnosis of health status. Among the concerns related to aging, cerebral atrophy, which leads to cognitive decline, is a substantial risk to the individual well-being, constituting a major public health burden. Brain volume loss is also associated with neurodegenerative diseases such as Alzheimer's disease and Parkinson's disease $[1,2]$.

The aging process in different brain regions can be detected through structural and functional Magnetic Resonance Imaging (MRI). As large-scale datasets such as UK Biobank that contain neuroimaging data are becoming readily available, there have been efforts to accurately predict an individual's chronological age with the neuroimaging datasets. Franke et al. [3] used principal component analysis and relevance vector machine to predict age reliably. Studies since then primarily used neural network models for data-driven feature extraction going beyond an arbitrary selection of features [4-11]. The convolutional neural network (CNN) models have been used with a satisfactory level of accuracy. The mean absolute error of the prediction in most literature with CNN models is between 2.14 and 3.4 years.

The difference between the predicted age and the actual chronological age, called delta age, has been used as an aging biomarker [3, 4]. After estimating the delta age, phenome-wide and genome-wide association tests have been applied to identify significantly associated genetic and clinical factors. Recent studies have shown that bone mineral density, blood pressure, and type 2 diabetes are associated with delta age [6, 7]. Genome-wide association analyses have demonstrated that KANSL1, MAPT-AS1, CRHR1, NSF in chromosome 17, KLF3 (chromosome 4), RUNX2 (chromosome 6), and NKX6-2 gene (chromosome 10) were significantly associated $[5,9,10]$. When combined with the cognitive test results, SNPs in MED8, COLEC10, and PLIN4 genes were also associated [10].

This study aimed to provide an explainable and causal basis of high delta age using multimodal data in UK Biobank. Our analysis includes structural MRI, genotype data from DNA array and whole-exome sequencing (WES), and phenotypes related to metabolomics and blood from UK Biobank. While existing research on delta age has focused on prediction methods and finding associations with other phenotypes, we identified which groups of biomarkers causally affect the degree of brain aging. In addition, we used an explainable Al method for medical images to identify brain regions that drive high delta age. 


\section{Results}

\section{Overview of the Analysis}

Figure 1 provides an overview of our analysis. First, a 3D CNN model was trained for age prediction with the T1-weighted structural brain MRI of healthy white British samples in the UK Biobank, excluding individuals with diseases related to cancer, diabetes, dementia, etc. The training was conducted via cross-validation to use all available samples in the downstream analysis. The Integrated Gradients (IG) method was then used to identify an accurate attribution of each voxel (volume + pixel) to the prediction [12]. Second, genome-wide association tests were conducted on different test levels to uncover novel loci associated with brain aging. We applied SAIGE (Scalable and Accurate Implementation of GEneralized mixed model) and SKAT-CommonRare (Sequence Kernel Association Test) methods for single variant-level and gene-level tests $[13,14]$. Lastly, we examined linear and nonlinear causal relationships between the delta age and the phenotypes (metabolomics and blood) with Mendelian randomization methods. The number of samples used in each step is in Table 1.

\section{Age Prediction Accuracy and Saliency Map}

Table 2 and Figure 2 show the prediction results of the 3D CNN model. Table 2 shows the mean absolute error of samples with diseases, samples without diseases, and four-fold cross-validation groups (see Methods). The mean absolute error in the healthy individuals was about 2.6406 years in the test set and 0.8989 years in the training set. The existing studies on brain age estimation had similar accuracy to this result [6-9]. The error in the samples with diseases was 2.651 years. Figure 2 (a) shows the correlation between the chronological age ( $x$-axis) and the predicted age ( $y$-axis). The chronological age and the predicted age showed a positive correlation. The age-related bias was replicated, showing delta age values biased toward the mean, with higher values for the younger samples (Figure 2 (b)) [15]. The bias was corrected by linear regression on the chronological age. Figure 2 (c) is a scatter plot between the chronological age and the adjusted delta age.

Figure 3 shows the saliency maps of the age prediction model with integrated gradients. Absolute values of integrated gradients were averaged for 100 samples with the youngest predicted age. Figure 3 (a) shows the voxels with averaged integrated gradients greater than five from the models trained with corresponding cross-validation batches. The gradients from the models had similar patterns to one another. The points were concentrated in the central part of the brain. According to the Automated Anatomical Labeling atlas (AAL) and the Natbrainlab atlas, when highlighting the regions with integrated gradients greater than ten, the regions were the fornix and the lower part of the thalamus (Figure 3 (b)) [16, 17]. Similar results were shown when the 100 samples were chosen randomly or by descending order of the delta age value (data not shown). 
medRxiv preprint doi: https://doi.org/10.1101/2022.03.04.22271813; this version posted March 7, 2022. The copyright

holder for this preprint (which was not certified by peer review) is the author/funder, who has granted medRxiv a license to display the preprint in perpetuity.

It is made available under a CC-BY 4.0 International license.

\section{Genetic Variants Associated with Delta Age}

We performed genetic association analyses for delta age. We carried out singlevariant tests for 93 million array-genotyped and imputed genetic variants using SAIGE. Since the single-variant test for rare variants in WES has low power, we used a gene-based test method for WES [18]. We used SKAT-CommonRare to test for both common and rare variant associations simultaneously $(19,871$ genes).

Figure 4 (a) and (b) are the Manhattan plots of single-variant analysis results from array-genotyped and imputed data and gene-based test results from whole-exome sequencing (WES). Table 3 and Table 4 list significant variants and genes. The pvalue threshold in Table 4 was the Bonferroni corrected level of $0.05(0.05 / 19,871)$. The single-variant test result showed that five loci in chromosomes 1, 4, 6, 10, 17, and one variant in chromosome 11 were significantly associated with the delta age. The nearest genes were STX6, MR1, KLF3-AS1, WNT16, INPP5A, NKX6-2, and several genes in chromosome 17, including KANSL1, MAPT-AS1, and NSF. Genetic heritability calculated by the variance components in the SAIGE model was 21.5 percent. The gene-based SKAT-CommonRare test showed 21 significant genes. Among them, to the best of our knowledge, OMA1, NOSTRIN, and CPNE3 genes were not reported in the existing GWAS studies on brain delta age. When looking at the SNP-level significance of the corresponding genes, a few common variants drove the gene-level significance (Supplementary Figure 1 (a)). Those top common variants were not reported to be significant in the single-variant test, possibly due to low imputation quality since the R-squared values of the leading SNPs were low in Supplementary Figure 1 (b).

To check whether delta age was truly driven by voxel values in the fornix and the lower part of the thalamus, we carried out the same GWAS procedure with the average voxel value of the two regions. The SAIGE results showed that the significant loci associated with the two regions were also concentrated on chromosome 17 (Supplementary Table 1 and Supplementary Figure 2 (a), (b)). SLC39A8 and C16orf95 genes were commonly shown to be associated with the two regions. The SKAT-CommonRare results were similar to the result from delta age (Supplementary Table 2 and Supplementary Figure 2 (c)). Six gene regions (OMA1, NOSTRIN, CPNE3, SFMBT2, NT5C3B, and HIGD1B) were commonly shown to be significant regions in the three SKAT-CommonRare tests on delta age, fornix volume, and thalamic volume. We also calculated the genetic correlation among delta age and average voxel values of the two regions and observed high genetic correlations (Supplementary Table 3 ). Our analysis results clearly showed the shared genetic basis of delta age and two regions.

Additional validation on the delta age of 1,610 healthy non-British white samples was conducted. In single-SNP GWAS, two SNPs in chromosome 4 with no specific gene region and two SNPs in chromosome 17 (rs375822897 in PLEKHM1 and rs56303031 in LINC02210-CRHR1) had p-values less than 0.05. Due to the small sample size (905 samples), none of the genes in Table 4 had p-values $<0.05$ in non-British white samples. 


\section{Causal Biomarkers of Delta Age}

Among the 310 phenotypes (249 metabolomic phenotypes and 61 phenotypes related to blood), 59 had $p$-values less than 0.05 in causal estimates from at least one of the three linear MR methods (MR-Egger regression, inverse variance weighting, and weighted median). Table 5 lists four phenotypes that were significant in the MR-Egger regression after multiple testing correction (Eosinophil count, Eosinophil percentage, Neutrophil count, and Total protein), and Supplementary Table 4 shows the other 55 phenotypes that were not significant. The immune-related biomarkers were reported to causally increase delta age.

Figure 5 is a PheWAS plot of the causal estimates from the MR-Egger regression. Overall, phenotypes related to white blood cells showed more significant causal relationships with delta age than other phenotypes. Similar results were replicated by the weighted median method (Supplementary Figure 3 and Supplementary Table 5). The significant causal phenotypes in MR analysis differed from those in association analysis with linear regression for delta age as a dependent variable (Supplementary Figure 4 and Supplementary Table 6).

Nonlinear MR analysis using piecewise MR and kernel IV showed similar results. Total cholines (0.03413), total lipids in small LDL (0.03599), and cholesteryl esters to total lipids in very large HDL percentage (0.01002) passed the test of the assumptions for instrument variable regression and returned $p$-value less than 0.05 in the trend test, indicating that there was nonlinearity in the causal relationship between the biomarkers and delta age (the values in the parenthesis indicate the p-values). Total cholines showed a causal relationship with the threshold. It causally increases delta age when it is above $2.5 \mathrm{mmol} / \mathrm{l}$. The other two biomarkers showed an inverted U-shaped relationship (Supplementary Figure 5). However, when applying multiple testing corrections, none of the three biomarkers were significant. 


\section{Discussion}

In this paper, we have analyzed the risk factors of brain aging with multimodal data. The CNN model that predicts age from brain MRI had a decent accuracy with the mean absolute error of 2.64 years. Visual information of the regional importance in the brain was extracted from the neural network model. Genetic variants and biomarkers that have significant links to brain aging were identified using GWAS methods and Mendelian randomization.

We used integrated gradients to make accurate saliency maps by incorporating information from a wider range of pixel values not present in the original images. This information revealed important brain regions missed by other mapping methods. The saliency map in the previous studies highlighted the brain regions such as the hippocampus, brainstem, and amygdala $[6,7,19]$. When highlighting the important points with higher integrated gradients in our study, they were centered on the fornix and the lower part of the thalamus. This indicates that the aging process affects the brain mainly through the atrophy in the inner area connected to memory and learning ability [20-23]. In addition, genetic variants associated with volumes of these regions and delta age were highly similar, supporting this result.

Investigating gene-level associations in the WES data, we identified novel loci (OMA1, NOSTRIN, and CPNE3) associated with the delta age. The novel and other significant genes were related to carcinogenesis, immune response, and neuronal functions. Proteins encoded by CPNE3 and TTK genes have been associated with carcinogenesis [24-26]. NOSTRIN gene in chromosome 2 participates in nitric oxide synthase trafficking, where nitric oxide (NO) is a signaling molecule in neurotransmission and inflammatory response. Abnormal NO signaling contributes to pathologies such as Parkinson's disease and stroke [27]. OMA1 and HIGD1B serve roles in maintaining the mitochondrial system, which is vital in cell survival and delaying neuronal death $[28,29]$. In the single-variant test of array genotyped and imputed variants, we replicated strong association signals in chromosome 17. The significant markers in other chromosomes were in STX6, MR1, KLF3-AS1, WNT16, INPP5A, and NKX6-2 genes. STX6 and KLF3-AS1 relate to carcinogenesis $[30,31]$. MR1 and WNT16 participate in immune response via antigen presentation to $T$ cells and lymphocyte proliferation [32, 33]. Mutations in INPP5A and NKX6-2 were shown to cause neurologic problems [34, 35].

We investigated the causal effects of all 310 blood and metabolomic biomarkers on delta age (249 metabolomic phenotypes and 61 phenotypes related to blood). The significant biomarkers from the MR-Egger regression and the weighted median method showed the potential causal roles of immune responses to delta age. Especially, biomarkers related to white blood cells had a significant causal relationship with delta age. This claim is supported by existing literature $[36,37]$ 
medRxiv preprint doi: https://doi.org/10.1101/2022.03.04.22271813; this version posted March 7, 2022. The copyright

holder for this preprint (which was not certified by peer review) is the author/funder, who has granted medRxiv a license to display the preprint in perpetuity.

It is made available under a CC-BY 4.0 International license .

This study, however, is subject to some limitations. First, the analysis was done in the European ancestry group only. The aging process and genetic variants associated with aging can differ according to ancestry. Second, replication of the results in an independent dataset was not conducted due to a lack of datasets with both genotype and brain MRI. Future research should focus on addressing the limits and making results more generalizable.

In conclusion, our multimodal data analysis shows many aspects of brain aging, including brain regions most affected by the aging, associate genes, and causal biomarkers. As more biobanks with multimodal data are collected, more diverse aspects of brain aging can be revealed. Biobanks of other ancestry groups can identify novel genetic biomarkers associated with brain aging not found in this study. Plus, potential mediating factors between immune responses and brain aging can be identified. This would allow a deeper understanding of brain aging mechanisms that develop proper medication and post-treatment. 


\section{Methods}

\section{Data Preprocessing}

T1-weighted structural MRI images of 34,129 white British samples in the UK Biobank were used for the analysis to minimize the effect of ancestry (average age of 60.964) [38]. All of the images downloaded from the UK Biobank had been normalized into MNI152 space (Montreal Neurosciences Institute) to render the comparison of each voxel possible [39]. The samples were selected if they had proper images and if they had no relation to any other individuals in the dataset. Each image was resized from $182 \times 216 \times 182$ to $128 \times 128 \times 128$ to reduce the computation cost. First, a part of z-axis voxels (from 26 to 153 out of 182 points) was selected to include various brain regions in the prediction task. The voxels in the upper outermost surface of the brain were excluded since they were considered negligible in the prediction and redundant due to the inclusion of other parts of the cerebral cortex. Second, each 2-dimensional 182x216 image ( $x$, $y$-axis) in z-axis points was resized to a $128 \times 128$ image with the nearest neighbors scaling algorithm. The preprocessing of nifti format MRI images was performed with oro.nifti and OpenlmageR packages in $R[40,41]$.

The 249 metabolomic phenotypes and 61 biomarkers from blood assays and blood count were used in the Mendelian randomization (MR) analysis. These were collected separately from MRI imaging, from 2006 to 2010; this would enable the investigation of the biomarkers' longitudinal and cumulative effect on the brain. The missing values in the selected biomarkers were imputed with multiple imputation by chained equations (MICE) to fit the missing values to the overall multivariate distribution [42]. After filling the missing values, 8,464 individuals with the brain MRI had corresponding values of metabolomic phenotypes, and all 34,129 individuals had values of blood-related phenotypes. The 310 selected biomarkers were divided into 17 groups, including amino acids, cholesterol, and glycolysis-related metabolites.

\section{D CNN Prediction Model and Integrated Gradients}

3D CNN model was used to predict the age of the individuals (Keras/Tensorflow library version 2.0 with Nvidia Titan RTX GPU). Supplementary Figure 6 is the overall network structure of the prediction model. The neural network model takes the resized images $(128 \times 128 \times 128)$ as input and has less than three million parameters to train. The spatial dropout layers were added in the first two blocks to prevent the model from overfitting. The kernel size is $3 \times 3 \times 3$ in convolution layers and $2 \times 2 \times 2$ in pooling layers. The model conducts max-pooling until the size of an image in each feature becomes $2 \times 2 \times 2$. The number of features increases as the input image size reduces. Adam optimizer with learning rate 0.001 and He uniform initializer were used since the activation function is the rectified linear unit (ReLU). 
Healthy white British individuals $(25,656)$ were selected to train the prediction model. Individuals with diseases (all types of cancers, diabetes, neoplasm, dementia, and mental disorders) were excluded from the training process. The dataset with healthy individuals was divided into four sets (CV1, CV2, CV3, and CV4) for four-fold cross-validation so that every sample is included in the test set at least once and has a predicted age value. When CV1 is the test dataset, the other three sets become the training dataset. Using the training set, three separate models (the same structure in Supplementary Figure 6 with different initial weights and dropouts) were trained for more robust prediction. They constitute a single model set. After training the models, the test images were given to the models as input. The average of the predictions from the three models becomes the final predicted age of the test images, hence a total of 12 models to train (three models for each of the four cross-validation batches). Prediction of the age of individuals with diseases was made with the average of the predicted age from the four trained model sets. The delta age value of each sample was calculated by subtracting the individual's chronological age from the predicted age. The age-related bias in the delta age value was adjusted through linear regression on the chronological age. The adjusted delta age values were used in the later association tests.

To identify which part of the brain contributes significantly to age prediction, the Integrated Gradients (IG) method was used. The IG method is an explainable AI method for neural networks that uses multiple images between blank and original images [12]. In this study, the number of images generated for each sample was 101 in reference to the recommended step size in the original paper.

\section{Genome-wide Association Test with Single Variants and Gene Regions}

93 million Array+Imputation SNPs were tested with SAIGE, and 8 million wholeexome sequencing (WES) markers were tested with the SKAT-CommonRare for association with delta age. SAIGE adopts a mixed effect model to deal with cryptic relatedness between the individuals. SKAT-CommonRare is a gene-based test of both common and rare variants $[13,14]$. Since the number of tests decreases in the gene-based test, multiple testing correction is less stringent.

$34,129(=N)$ samples were used in SAIGE analysis. The delta age values of the individuals were inverse-normal transformed. Covariates were sex, age, ten principal component scores, and four dummy variables which indicate different cross-validation test sets plus samples with diseases. $N \times N$ genomic relation matrix (GRM) was calculated by 784,256 markers in called autosomal genotypes. Leave-one-chromosome-out (LOCO) option was applied when estimating the GRM.

19,871 regions were identified with annotation on the WES genotype by the ANNOVAR software [43]. The size of the samples $n$ in the gene-based test was 19,057 (white British individuals with proper MRI images included in the UK Biobank 200k whole-exome sequencing data). The same covariates in the SAIGE 
test were used. The threshold minor allele frequency for rare variants was $\frac{1}{\sqrt{2 n}}$ (0.0051). The beta weights given to the variance of effect size were set to beta $(1,25)$ for rare variants and beta $(0.5,0.5)$ for the common variants.

Genetic correlation among delta age and the average volume of two brain regions (the fornix and the lower part of the thalamus) was derived using LD score regression with western European LD scores [44].

\section{Linear and Nonlinear Mendelian Randomization}

We used variations of Mendelian randomization methods to identify the causal effect of biomarkers (exposure) on delta age (outcome). The overall workflow of the Mendelian randomization in this study is in Supplementary Figure 7. Instrument genetic markers for each of the 310 biomarkers were selected as follows. First, we chose variants significantly associated with the exposure ( $p$-value under 5e-8) among the called autosomal genetic markers. The markers with minor allele frequencies less than 0.01 were pruned because the estimation of the effect size from rare variants is unstable. The GWAS summary statistics for the metabolomics measured by the Nightingale Health are from open datasets in MRC Integrative Epidemiology Unit at the University of Bristol (IEU) [45]. The SAIGE GWAS results of blood phenotypes are from Pan-UK Biobank GWAS summary statistics by the Broad Institute (available at https://pan.ukbb.broadinstitute.org). We used effect size, standard error, and p-value from the samples with European ancestry. Second, the linkage disequilibrium (LD) pruning process was conducted with PLINK software with a window size of 50 base pairs to ensure that the selected instruments were independent of each other [46]. Pairs of variants with a correlation coefficient larger than 0.01 were LD pruned. Lastly, since the markers should not directly affect the outcome, variants with $p$-value with regard to the outcome less than 0.05 divided by the number of markers left were excluded. The effect size and standard error of the remaining markers were used in the MR analysis.

\section{Linear Exposure-Outcome Relationship}

We first calculated the causal impacts of the biomarkers (exposure) on delta age (outcome) with three linear Mendelian randomization methods: MR-Egger regression, weighted median method (WM), and inverse variance weighting method (IVW) [47-49]. The MendelianRandomization package in $\mathrm{R}$ was used to carry out variations of the MR-Egger regression [50]. The estimates and $p$-values from the default version of MR-Egger regression were selected. The same was done for the estimates of the WM and IVW method. The estimates in all methods were assumed to follow the normal distribution. The Benjamini-Hochberg procedure was applied to control the false discovery rates [51]. 


\section{Adding Nonlinearity}

Nonlinear MR was conducted with the nlmr package in $\mathrm{R}$ [52]. The key rationale for using the nonlinear Mendelian randomization method is to find a nonlinear pattern of causal estimates from different ranges of exposure, as the impact of exposure on delta age can vary according to the ranges.

The assumptions for instrument variable regression were first thoroughly tested. Among the instruments selected for each biomarker from the linear MR procedure, the ones with positive effect sizes were collected. We constructed each sample's single allele score $G$ with those markers. Each marker has genotype 0, 1, or 2 for each sample. When there are $n$ samples and $m$ genetic markers, the allele score of sample $i$ is in (1).

$$
G_{i}=\sum_{j=1}^{m} \beta_{j} g_{i j}
$$

Here $\beta_{j}$ is the effect size of the $j$ th marker and $g_{i j}$ is the genotype of sample $i$ in the $j$ th marker. The allele scores and the exposure values were tested for a significant positive relationship ( $p$-value of the Pearson's correlation coefficient lower than 0.05 using the cor.test function in R). Exclusion restriction was assumed to be testable with conditional independence tests ( $Y$ and $G$ conditionally independent given $X$ ) because the directions of effect between $G, X$, and $Y$ were fixed ( $G \rightarrow X \rightarrow Y$ ). The allele score shows a genetically determined level of the biomarker, and the delta age was calculated from images taken after the level of the biomarker had been measured. In order to test for nonlinear conditional independence, Randomized Conditional Independence Test (RCIT) was used [53]. We repeated the RCIT three times. The assumptions were considered to have been met if none of the three tests had a p-value less than 0.05 with the null hypothesis of the conditional independence between $Y$ and $G$ given $X$. Only 12 out of 310 variables were found to satisfy all the assumptions for instrument variable regression.

Then, we conducted the piecewise MR analysis for the 12 variables that met the assumptions [52]. The samples were divided into ten groups according to deciles by the IV-free exposure. Assumptions of the IV-exposure relationship in the piecewise MR are the homogeneity and the linearity across all samples. These assumptions were tested by the heterogeneity test using $Q$ statistics. If the null hypothesis of homogeneity in the estimates between the groups was not rejected in the heterogeneity test, the trend test was conducted on the biomarker. The trend test looked at whether the local average causal effect in each group is significantly explained by the average value of the exposure in the corresponding group. Kernel IV regression with radial basis function kernel was performed with the 12 passed phenotypes to see if the results would be replicated. Here 2,000 people were sampled because heavy computation is required for an inverse matrix of a large matrix. The test values were 1,000 numbers with an equal distance between the minimum and the maximum of the exposure values. 
medRxiv preprint doi: https://doi.org/10.1101/2022.03.04.22271813; this version posted March 7, 2022. The copyright

holder for this preprint (which was not certified by peer review) is the author/funder, who has granted medRxiv a license to display the preprint in perpetuity.

It is made available under a CC-BY 4.0 International license.

\section{Data Availability}

The summary statistics used in the Mendelian randomization analysis are available for public download at the IEU OpenGWAS Project (https://gwas.mrcieu.ac.uk) and the Pan-UK Biobank (https://pan.ukbb.broadinstitute.org).

\section{Code Availability}

The code used in the analyses is available at our Github page.

https://github.com/Flumenlucidum/Brain-Aging.

We used publicly available software for the analyses.

MICE: https://github.com/amices/mice

TensorFlow: https://www.tensorflow.org

SAIGE: https://github.com/weizhouUMICH/SAIGE

SKAT: https://github.com/leelabsg/SKAT

LDSC: $\underline{\text { https://github.com/bulik/ldsc }}$

PLINK2: https://www.cog-genomics.org/plink/2.0

MendelianRandomization: https://github.com/cran/MendelianRandomization

nlmr: https://github.com/jrs95/nlmr

\section{Acknowledgements}

This research was supported by Big Brain Project through the National Research Foundation of Korea (NRF) funded by the Ministry of Science and ICT (No. 2021M3E5D2A0102249311), and the Brain Pool Plus (BP+, Brain Pool+) Program through the National Research Foundation of Korea (NRF) funded by the Ministry of Science and ICT (2020H1D3A2A03100666). UK Biobank data were accessed under the accession number UKB: 45227.

\section{Author Contributions}

J.K. and S.L. designed the experiments. J.K., J.L., and S.L. constructed and developed the age prediction model. J.K. and S.L. wrote the manuscript. All authors reviewed and approved the final version of the manuscript. 
medRxiv preprint doi: https://doi.org/10.1101/2022.03.04.22271813; this version posted March 7, 2022. The copyright holder for this preprint (which was not certified by peer review) is the author/funder, who has granted medRxiv a license to display the preprint in perpetuity.

It is made available under a CC-BY 4.0 International license .
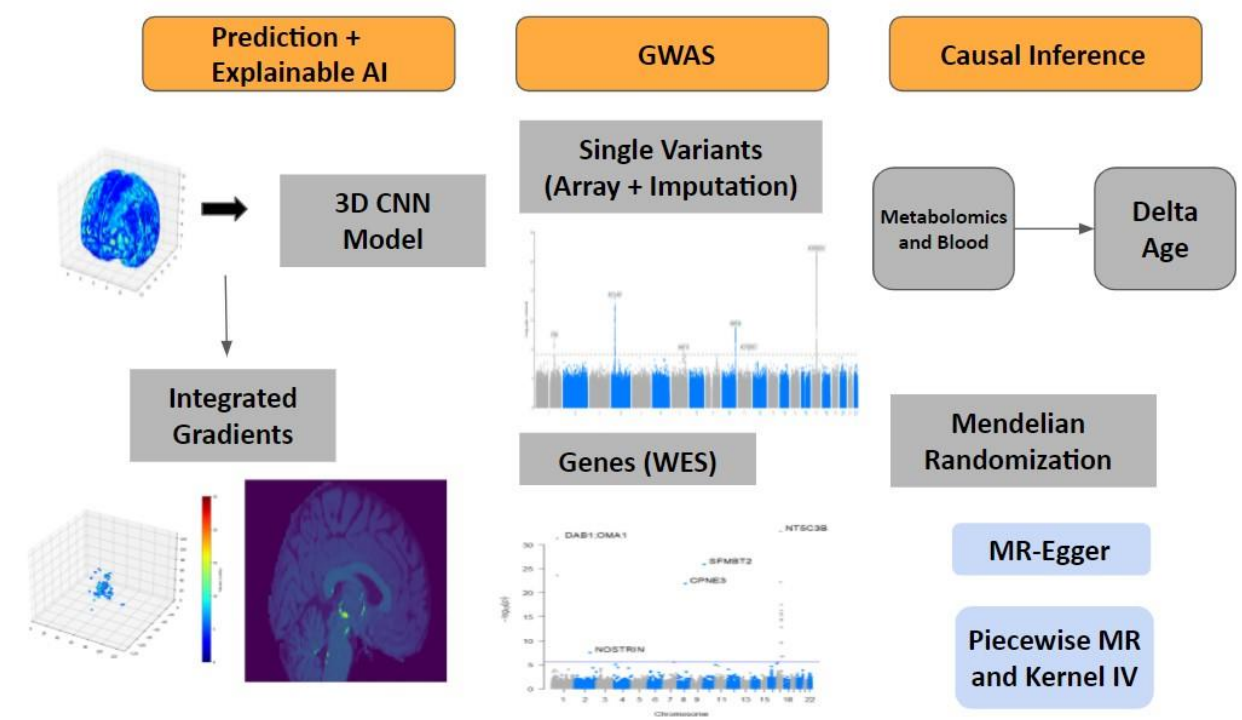

Mendelian

Randomization

Figure 1 Overview of the analysis 
medRxiv preprint doi: https://doi.org/10.1101/2022.03.04.22271813; this version posted March 7, 2022. The copyright holder for this preprint (which was not certified by peer review) is the author/funder, who has granted medRxiv a license to display the preprint in perpetuity.

It is made available under a CC-BY 4.0 International license.

(a)

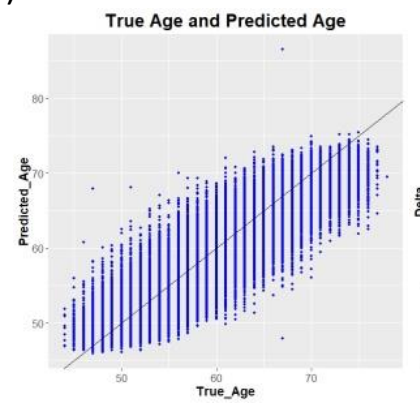

(b)

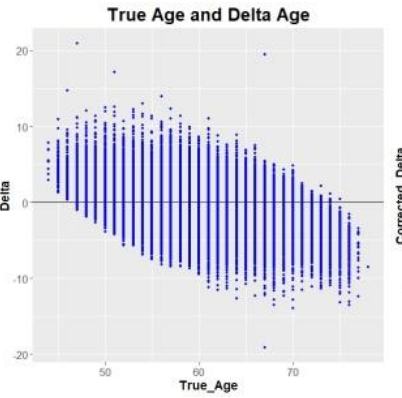

(c) True Age and Corrected Delta Age

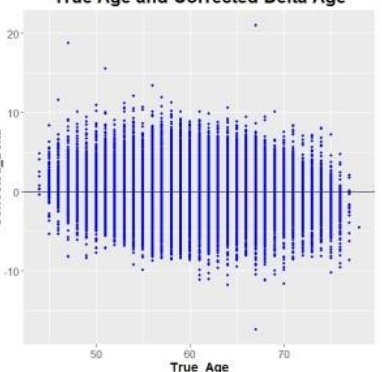

Figure 2 Scatterplots of chronological age (x-axis) with (a) predicted age, (b) delta age, and (c) adjusted delta age ( $y$-axis) 
medRxiv preprint doi: https://doi.org/10.1101/2022.03.04.22271813; this version posted March 7, 2022. The copyright holder for this preprint (which was not certified by peer review) is the author/funder, who has granted medRxiv a license to display the preprint in perpetuity.

It is made available under a CC-BY 4.0 International license.

(a)
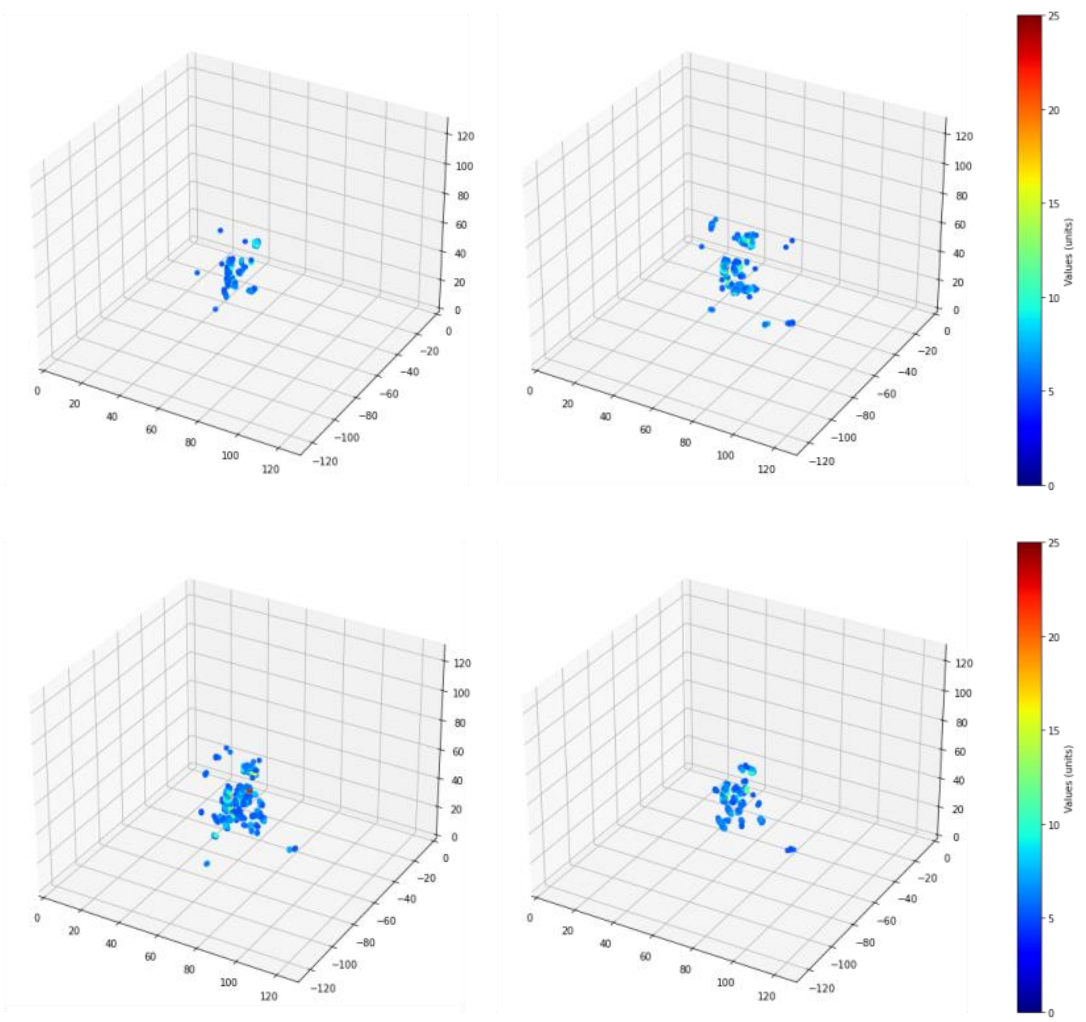

(b)

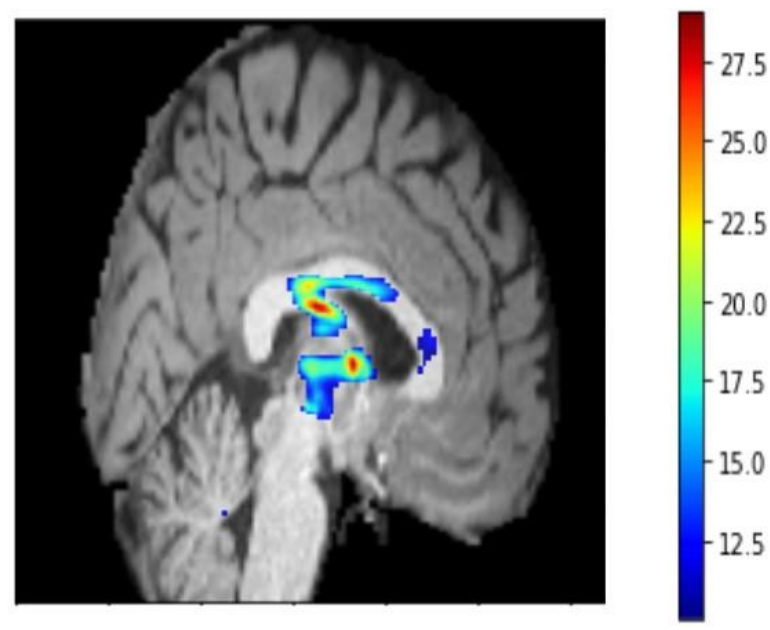

Figure 3 (a) The points with absolute IG greater than five in four CV model sets extracted from 100 individuals with the youngest predicted age (b) We derived the IG again (with different dropouts) on the CV2 batch. Voxels with absolute IG greater than ten concentrated on the fornix and the lower part of the thalamus (the x-coordinate of 62). 
medRxiv preprint doi: https://doi.org/10.1101/2022.03.04.22271813; this version posted March 7, 2022. The copyright holder for this preprint (which was not certified by peer review) is the author/funder, who has granted medRxiv a license to display the preprint in perpetuity.

It is made available under a CC-BY 4.0 International license.

(a) SAIGE result on delta age (single-variant test with array-genotyped and imputed data)

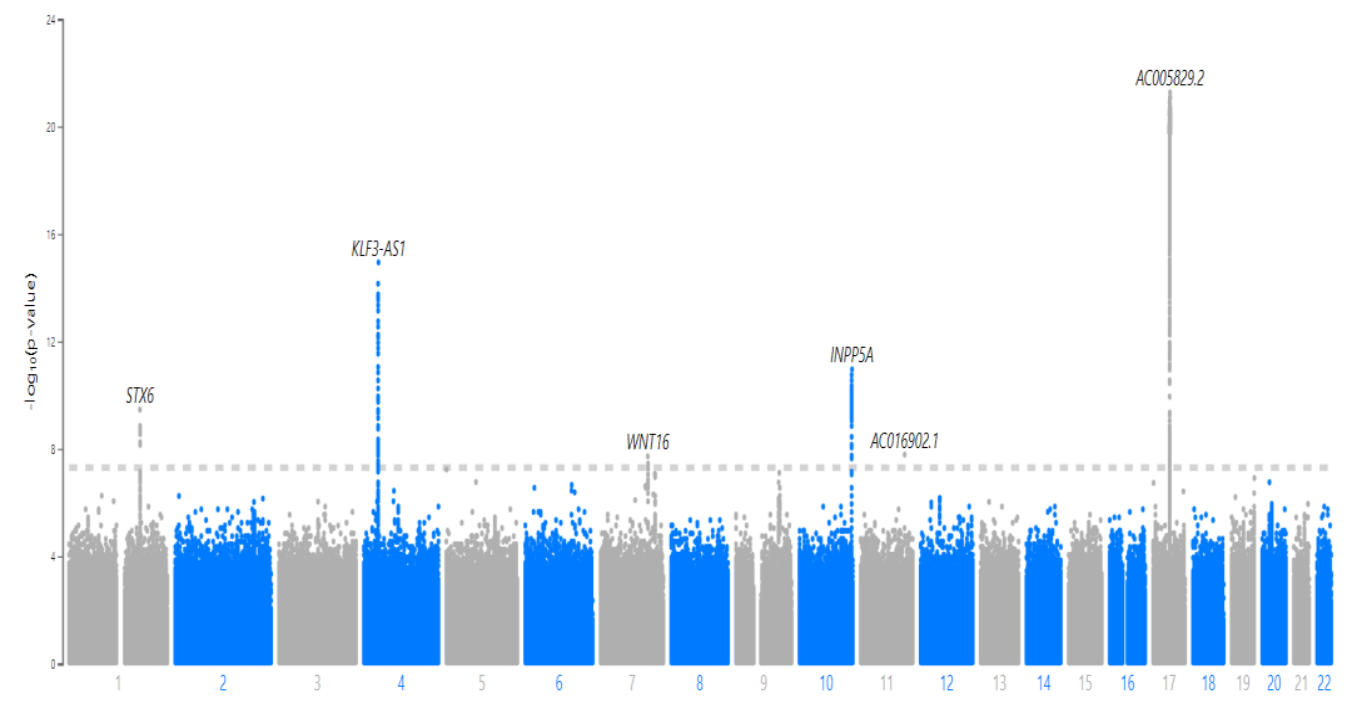

(b) SKAT-CommonRare result on the fornix volume (gene-based test with WES data)

NT5C3B

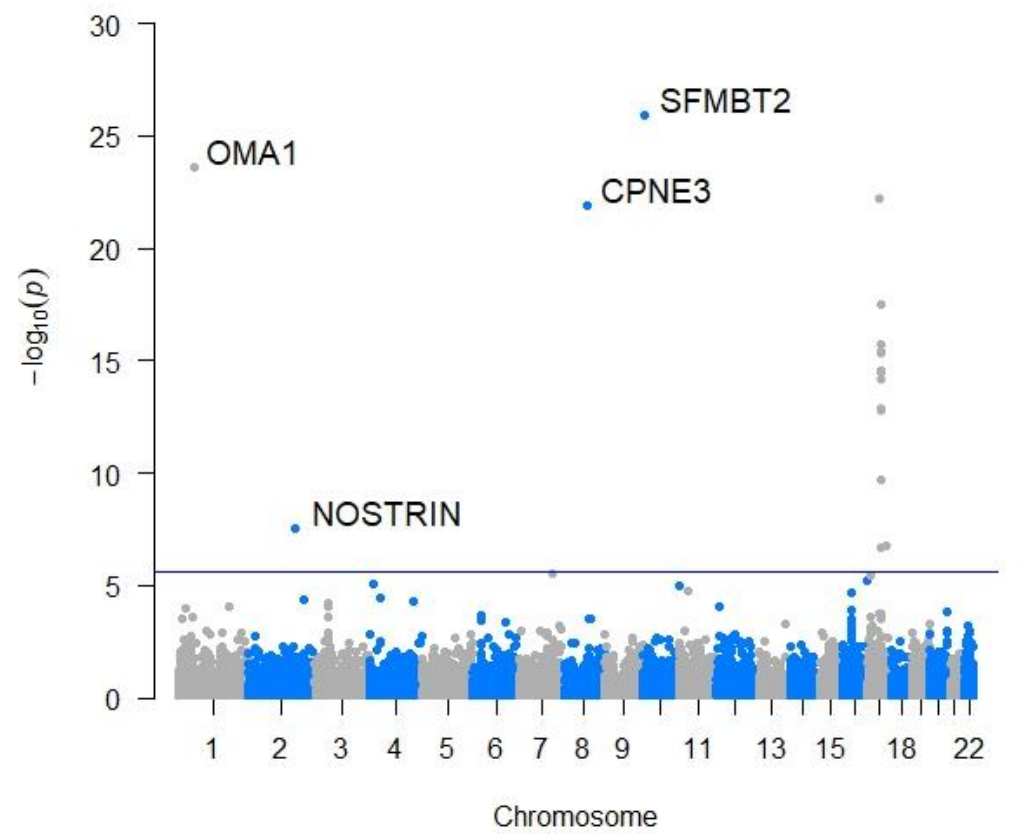

Figure 4 Manhattan plots of (a) SAIGE and (b) SKAT-CommonRare result on delta age 
medRxiv preprint doi: https://doi.org/10.1101/2022.03.04.22271813; this version posted March 7, 2022. The copyright holder for this preprint (which was not certified by peer review) is the author/funder, who has granted medRxiv a license to display the preprint in perpetuity.

It is made available under a CC-BY 4.0 International license .

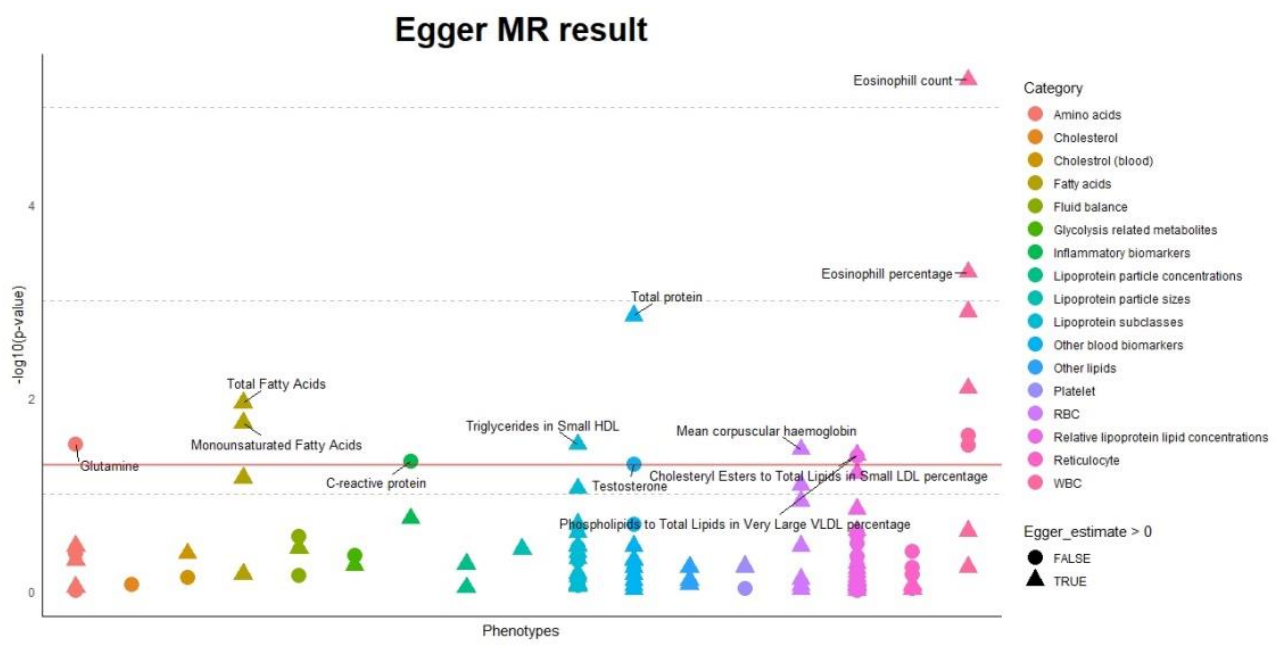

Figure 5 A PheWAS plot of Linear MR causal estimates from MR-Egger regression. The horizontal red line is the $p$-value threshold of 0.05 . Each color indicates a different group of phenotypes. The two most significant variables in each group with p-values under 0.05 were labeled in each plot. The shape of the points shows the direction of the causal effect. Triangles are biomarkers that contribute to higher delta age, and circles are biomarkers that contribute to lower delta age. 
medRxiv preprint doi: https://doi.org/10.1101/2022.03.04.22271813; this version posted March 7, 2022. The copyright holder for this preprint (which was not certified by peer review) is the author/funder, who has granted medRxiv a license to display the preprint in perpetuity.

It is made available under a CC-BY 4.0 International license.

Table1 The number of samples in each stage of analysis

\begin{tabular}{|l|l|l|l|l|l|l|}
\hline $\begin{array}{l}\text { 3D CNN } \\
\text { (Training) }\end{array}$ & $\begin{array}{l}\text { 3D CNN } \\
\text { (Test) }\end{array}$ & IG & $\begin{array}{l}\text { GWAS } \\
\text { (SNPs) }\end{array}$ & $\begin{array}{l}\text { GWAS } \\
\text { (WES) }\end{array}$ & $\begin{array}{l}\text { MR } \\
\text { (Metabolo } \\
\text { mics) }\end{array}$ & $\begin{array}{l}\text { MR } \\
\text { (Blood) }\end{array}$ \\
\hline 25,656 & 34,129 & 34,129 & 34,129 & 19,057 & 8,464 & 34,129 \\
\hline
\end{tabular}

Table 2 The mean absolute error (MAE) of individuals without diseases, each cross-validation batch, and individuals with diseases

\begin{tabular}{|l|l|l|l|l|l|l|}
\hline & Overall & CV1 & CV2 & CV3 & CV4 & Disease \\
\hline Training set & $\mathbf{0 . 8 9 8 9}$ & 0.7801 & 0.8948 & 0.9900 & 0.9309 & - \\
\hline Test set & $\mathbf{2 . 6 4 0 6}$ & 2.6226 & 2.7113 & 2.6351 & 2.5933 & 2.6510 \\
\hline
\end{tabular}

Table 3 SNPs in top loci associated with delta age (SAIGE, single-variant test with array-genotyped and imputed data)

\begin{tabular}{|l|l|l|l|l|l|}
\hline Chromosome & Location & rsID (GRCh37) & Nearest gene & Effect size & P-value \\
\hline 17 & $44,335,635$ & rs147431626 & AC005829.2 & 0.0875 & $5.36 \mathrm{E}-22$ \\
\hline 4 & $38,638,966$ & rs201791735 & KLF3-AS1 & 0.0661 & $1.14 \mathrm{E}-15$ \\
\hline 10 & $134,559,486$ & - & INPP5A & 0.0607 & $1.10 \mathrm{E}-11$ \\
\hline 1 & $180,963,000$ & rs534102361 & STX6 & -0.0480 & $3.46 \mathrm{E}-10$ \\
\hline 11 & $112,766,643$ & rs536165403 & AC016902.1 & 1.7857 & $1.64 \mathrm{E}-08$ \\
\hline 7 & $120,981,641$ & - & WNT16 & -0.0438 & $1.90 \mathrm{E}-08$ \\
\hline
\end{tabular}

Table 4 Gene regions associated with delta age (SKAT-CommonRare, gene-based test with WES)

\begin{tabular}{|l|l|l|l|}
\hline Chromosome & Starting location (GRCh38) & Gene & P-value \\
\hline 17 & 41825424 & NT5C3B & $1.59 \mathrm{E}-33$ \\
\hline 10 & 7163670 & SFMBT2 & $1.27 \mathrm{E}-26$ \\
\hline 1 & 58480654 & OMA1 & $2.68 \mathrm{E}-24$ \\
\hline 17 & 44848057 & HIGD1B & $5.72 \mathrm{E}-23$ \\
\hline 8 & 86528449 & CPNE3 & $1.31 \mathrm{E}-22$ \\
\hline 17 & 46626669 & NSF & $3.12 \mathrm{E}-18$ \\
\hline 17 & 45844904 & SPPL2C & $1.99 \mathrm{E}-16$ \\
\hline 17 & 46031383 & KANSL1 & $4.15 \mathrm{E}-16$ \\
\hline 17 & 45962240 & MAPT & $4.26 \mathrm{E}-16$ \\
\hline
\end{tabular}


medRxiv preprint doi: https://doi.org/10.1101/2022.03.04.22271813; this version posted March 7, 2022. The copyright holder for this preprint (which was not certified by peer review) is the author/funder, who has granted medRxiv a license to display the preprint in perpetuity.

It is made available under a CC-BY 4.0 International license.

\begin{tabular}{|l|l|l|l|}
\hline 17 & 45437760 & PLEKHM1 & $2.95 \mathrm{E}-15$ \\
\hline 17 & 45999252 & STH & $3.56 \mathrm{E}-15$ \\
\hline 17 & 45395365 & ARHGAP27 & $7.11 \mathrm{E}-15$ \\
\hline 17 & 46540188 & LRRC37A2 & $1.34 \mathrm{E}-13$ \\
\hline 17 & 45834768 & LINC02210-CRHR1 & $1.53 \mathrm{E}-13$ \\
\hline 17 & 45844810 & MAPT-AS1 & $1.85 \mathrm{E}-10$ \\
\hline 2 & 168802560 & NOSTRIN & $3.03 \mathrm{E}-08$ \\
\hline 17 & 64854499 & LRRC37A3 & $1.71 \mathrm{E}-07$ \\
\hline 17 & 46553114 & ARL17A & $2.00 \mathrm{E}-07$ \\
\hline
\end{tabular}

Table 5 A list of causal estimates and p-values of significant biomarkers in the MREgger regression

\begin{tabular}{|l|l|l|}
\hline Biomarker & Estimates & P-value \\
\hline Eosinophil count & 0.151 & $5.16 \mathrm{E}-06$ \\
\hline Eosinophil percentage & 0.156 & $5.04 \mathrm{E}-04$ \\
\hline Neutrophil count & 0.153 & $1.28 \mathrm{E}-03$ \\
\hline Total protein & 0.181 & $1.42 \mathrm{E}-03$ \\
\hline
\end{tabular}


medRxiv preprint doi: https://doi.org/10.1101/2022.03.04.22271813; this version posted March 7, 2022. The copyright

holder for this preprint (which was not certified by peer review) is the author/funder, who has granted medRxiv a license to display the preprint in perpetuity.

It is made available under a CC-BY 4.0 International license.

\section{Bibliography}

[1] N. C. Fox and J. M. Schott, "Imaging cerebral atrophy: normal ageing to Alzheimer's disease," The Lancet, vol. 363, no. 9406, pp. 392-394, 2004.

[2] A. Nagano-Saito et al., "Cerebral atrophy and its relation to cognitive impairment in Parkinson disease," Neurology, vol. 64, no. 2, pp. 224-229, 2005.

[3] K. Franke, G. Ziegler, S. KI KI5.on to cognitive impairment in Parkinson disease," disease," ," $d$ in each plot. The shape of the points showsing kernel methods: exploring the influence of various parameters," Neuroimage, vol. 50, no. 3, pp. 883-892, 2010.

[4] J. H. Cole et al., "Predicting brain age with deep learning from raw imaging data results in a reliable and heritable biomarker," Neurolmage, vol. 163, pp. 115-124, 2017.

[5] B. A. J. Jp. et al., "Brain age prediction using deep learning uncovers associated sequence variants," Nature communications, vol. 10, no. 1, pp. 1-10, 2019.

[6] A. Kolbeinsson et al., "Accelerated MRI-predicted brain ageing and its associations with cardiometabolic and brain disorders," Scientific Reports, vol. 10, no. 1, pp. 1-9, 2020.

[7] N. K. Dinsdale et al., "Learning patterns of the ageing brain in MRI using deep convolutional networks," Neuroimage, vol. 224, p. 117401, 2021.

[8] H. Peng, W. Gong, C. F. Beckmann, A. Vedaldi, and S. M. Smith, "Accurate brain age prediction with lightweight deep neural networks," Medical image analysis, vol. 68, p. 101871, 2021.

[9] K. Ning et al., "Improving brain age estimates with deep learning leads to identification of novel genetic factors associated with brain aging," Neurobiology of Aging, vol. 105, pp. 199-204, 2021.

[10] A. Le Goallec, S. Diai, S. Collin, T. Vincent, and C. J. Patel, "Using deep learning to predict brain age from brain magnetic resonance images and cognitive tests reveals that anatomical and functional brain aging are phenotypically and genetically distinct," medRxiv, 2021.

[11] P. K. Lam et al., "Accurate brain age prediction using recurrent slice-based networks," in 16th International Symposium on Medical Information Processing and Analysis, 2020, vol. 11583: International Society for Optics and Photonics, p. 1158303.

[12] M. Sundararajan, A. Taly, and Q. Yan, "Axiomatic attribution for deep networks," in International Conference on Machine Learning, 2017: PMLR, pp. 3319-3328.

[13] W. Zhou et al., "Efficiently controlling for case-control imbalance and sample relatedness in large-scale genetic association studies," Nature genetics, vol. 50, no. 9, pp. 1335-1341, 2018.

[14] I. Ionita-Laza, S. Lee, V. Makarov, J. D. Buxbaum, and X. Lin, "Sequence kernel association tests for the combined effect of rare and common variants," The American Journal of Human Genetics, vol. 92, no. 6, pp. 841-853, 2013. 
[15] S. M. Smith, D. Vidaurre, F. Alfaro-Almagro, T. E. Nichols, and K. L. Miller, "Estimation of brain age delta from brain imaging," Neuroimage, vol. 200, pp. 528-539, 2019.

[16] N. Tzourio-Mazoyer et al., "Automated anatomical labeling of activations in SPM using a macroscopic anatomical parcellation of the MNI MRI single-subject brain," Neuroimage, vol. 15, no. 1, pp. 273-289, 2002.

[17] M. Catani and M. T. De Schotten, "A diffusion tensor imaging tractography atlas for virtual in vivo dissections," cortex, vol. 44, no. 8, pp. 1105-1132, 2008.

[18] S. Lee, G. R. Abecasis, M. Boehnke, and X. Lin, "Rare-variant association analysis: study designs and statistical tests," The American Journal of Human Genetics, vol. 95, no. 1, pp. 5-23, 2014.

[19] K.-M. Bintsi, V. Baltatzis, A. Hammers, and D. Rueckert, "Voxel-level Importance Maps for Interpretable Brain Age Estimation," in Interpretability of Machine Intelligence in Medical Image Computing, and Topological Data Analysis and Its Applications for Medical Data: Springer, 2021, pp. 65-74.

[20] W. Deeb et al., "Fornix-region deep brain stimulatione in Medical Image Computing, and Topological Data ANew England Journal of Medicine, vol. 381, no. 8, pp. 783-785, 2019.

[21] C. M. Foster, K. M. Kennedy, D. A. Hoagey, and K. M. Rodrigue, "The role of hippocampal subfield volume and fornix microstructure in episodic memory across the lifespan," Hippocampus, vol. 29, no. 12, pp. 1206-1223, 2019.

[22] A. Cherubini, P. PP. P06C. Caltagirone, U. Sabatini, and G. Spalletta, "Aging of subcortical nuclei: microstructural, mineralization and atrophy modifications measured in vivo using MRI," Neuroimage, vol. 48, no. 1, pp. 29-36, 2009.

[23] M. Wolff and S. D. Vann, "The cognitive thalamus as a gateway to mental representations," Journal of Neuroscience, vol. 39, no. 1, pp. 3-14, 2019.

[24] C. Heinrich et al., "Copine-III interacts with ErbB2 and promotes tumor cell migration," Oncogene, vol. 29, no. 11, pp. 1598-1610, 2010.

[25] B. Sun et al., "Circulating exosomal CPNE3 as a diagnostic and prognostic biomarker for colorectal cancer," Journal of cellular physiology, vol. 234, no. 2, pp. 1416-1425, 2019.

[26] C. Kasbek, C. H. Yang, and H. A. Fisk, "Mps1 as a link between centrosomes and genomic instability," Environmental and molecular mutagenesis, vol. 50, no. 8, pp. 654-665, 2009.

[27] J. R. Steinert, T. Chernova, and I. D. Forsythe, "Nitric oxide signaling in brain function, dysfunction, and dementia," The Neuroscientist, vol. 16, no. 4, pp. 435-452, 2010.

[28] Y. Pang et al., "HIGD-1B inhibits hypoxia-induced mitochondrial fragmentation by regulating OPA1 cleavage in cardiomyocytes," Molecular Medicine Reports, vol. 24, no. 2, pp. 1-11, 2021.

[29] A. Korwitz et al., "Loss of OMA1 delays neurodegeneration by preventing stress-induced OPA1 processing in mitochondria," Journal of Cell Biology, 
vol. 212, no. 2, pp. 157-166, 2016.

[30] J. Du, X. Liu, Y. Wu, J. Zhu, and Y. Tang, "Essential role of STX6 in esophageal squamous cell carcinoma growth and migration," Biochemical and biophysical research communications, vol. 472, no. 1, pp. 60-67, 2016.

[31] J.-Q. Liu et al., "IncRNA KLF3-AS1 suppresses cell migration and invasion in ESCC by impairing miR-185-5p-targeted KLF3 inhibition," Molecular Therapy-Nucleic Acids, vol. 20, pp. 231-241, 2020.

[32] G. De Libero, A. Chancellor, and L. Mori, "Antigen specificities and functional properties of MR1-restricted T cells," Molecular Immunology, vol. 130, pp. 148-153, 2021.

[33] J. Mazieres et al., "Inhibition of Wnt16 in human acute lymphoblastoid leukemia cells containing the $\mathrm{t}(1 ; 19)$ translocation induces apoptosis," Oncogene, vol. 24, no. 34, pp. 5396-5400, 2005.

[34] Q. Liu et al., "Cerebellum-enriched protein INPP5A contributes to selective neuropathology in mouse model of spinocerebellar ataxias type 17," Nature communications, vol. 11, no. 1, pp. 1-13, 2020.

[35] V. Chelban et al., "Genetic and phenotypic characterization of NKX6to selective neuropathology in mouse model of spEuropean journal of neurology, vol. 27, no. 2, pp. 334-342, 2020.

[36] C. Ising and M. T. Heneka, "Functional and structural damage of neurons by innate immune mechanisms during neurodegeneration," Cell death \& disease, vol. 9, no. 2, pp. 1-8, 2018.

[37] F. Corlier et al., "Systemic inflammation as a predictor of brain aging: Contributions of physical activity, metabolic risk, and genetic risk," Neuroimage, vol. 172, pp. 118-129, 2018.

[38] C. Bycroft et al., "The UK Biobank resource with deep phenotyping and genomic data," Nature, vol. 562, no. 7726, pp. 203-209, 2018.

[39] G. Grabner, A. L. Janke, M. M. Budge, D. Smith, J. Pruessner, and D. L. Collins, "Symmetric atlasing and model based segmentation: an application to the hippocampus in older adults," in International Conference on Medical Image Computing and Computer-Assisted Intervention, 2006: Springer, pp. 58-66.

[40] B. Whitcher, V. J. Schmid, and A. Thorton, "Working with the DICOM and NIfTI Data Standards in R," Journal of Statistical Software, vol. 44, no. 1, pp. 1-29, 2011.

[41] L. Mouselimis, "OpenImageR: An Image Processing Toolkit," $R$ package version, vol. 1, no. 5, 2017.

[42] I. R. White, P. Royston, and A. M. Wood, "Multiple imputation using chained equations: issues and guidance for practice," Statistics in medicine, vol. 30, no. 4, pp. 377-399, 2011.

[43] K. Wang, M. Li, and H. Hakonarson, "ANNOVAR: functional annotation of genetic variants from high-throughput sequencing data," Nucleic acids research, vol. 38, no. 16, pp. e164-e164, 2010.

[44] B. Bulik-Sullivan et al., "An atlas of genetic correlations across human diseases and traits," Nature genetics, vol. 47, no. 11, pp. 1236-1241, 2015.

[45] B. L. Elsworth et al., "The MRC IEU OpenGWAS data infrastructure," 
medRxiv preprint doi: https://doi.org/10.1101/2022.03.04.22271813; this version posted March 7, 2022. The copyright holder for this preprint (which was not certified by peer review) is the author/funder, who has granted medRxiv a license to display the preprint in perpetuity.

It is made available under a CC-BY 4.0 International license.

bioRxiv, 2020.

[46] S. Purcell et al., "PLINK: a tool set for whole-genome association and population-based linkage analyses," The American journal of human genetics, vol. 81, no. 3, pp. 559-575, 2007.

[47] S. Burgess, A. Butterworth, and S. G. Thompson, "Mendelian randomization analysis with multiple genetic variants using summarized data," Genetic epidemiology, vol. 37, no. 7, pp. 658-665, 2013.

[48] J. Bowden, G. Davey Smith, and S. Burgess, "Mendelian randomization with invalid instruments: effect estimation and bias detection through Egger regression," International journal of epidemiology, vol. 44, no. 2, pp. 512-525, 2015.

[49] J. Bowden, G. Davey Smith, P. C. Haycock, and S. Burgess, "Consistent estimation in Mendelian randomization with some invalid instruments using a weighted median estimator," Genetic epidemiology, vol. 40, no. 4, pp. 304-314, 2016.

[50] O. O. Yavorska and S. Burgess, "MendelianRandomization: an R package for performing Mendelian randomization analyses using summarized data," International journal of epidemiology, vol. 46, no. 6, pp. 1734-1739, 2017.

[51] Y. Benjamini and Y. Hochberg, "Controlling the false discovery rate: a practical and powerful approach to multiple testing," Journal of the Royal statistical society: series B (Methodological), vol. 57, no. 1, pp. 289-300, 1995.

[52] J. R. Staley and S. Burgess, "Semiparametric methods for estimation of a nonlinear exposuresurepproach to multiple testing," mmarized data," a," weighted median estimator," r," ts," in " Genetic epidemiology, vol. 41, no. 4, pp. 341-352, 2017.

[53] E. V. Strobl, K. Zhang, and S. Visweswaran, "Approximate kernel-based conditional independence tests for fast non-parametric causal discovery," Journal of Causal Inference, vol. 7, no. 1, 2019. 